\title{
Les origines théâtrales de la photographie
}

Dominique de Font-Réaulx

\section{(2) OpenEdition \\ Journals}

\section{Édition électronique}

URL : http://journals.openedition.org/shakespeare/3052

DOI : 10.4000/shakespeare.3052

ISSN : 2271-6424

Éditeur

Société Française Shakespeare

Référence électronique

Dominique de Font-Réaulx, «LeS ORIgines théâtrales de LA PHotograPhiE 》, Actes des congrès de la Société française Shakespeare [En ligne], 33 | 2015, mis en ligne le 10 mars 2015, consulté le 02 juin 2020. URL : http://journals.openedition.org/shakespeare/3052 ; DOI : https://doi.org/10.4000/

shakespeare.3052

Ce document a été généré automatiquement le 2 juin 2020.

(C) SFS 


\title{
Les origines théâtrales de la photographie
}

\author{
Dominique de Font-Réaulx
}

«Le troisième acte [du Songe, joué en 1818 à l'Ambigu-Comique] offre une décoration représentant un clair de lune que l'on peut considérer comme un chef d'œuvre du genre. Le succès en est dû au seul Daguerre et c'est lui seul qu'on devrait nommer ${ }^{1}$.» Jacques Louis Mandé Daguerre (1787-1851), inventeur en 1838 du daguerr-é-otype, premier procédé photographique à pouvoir être utilisé et développé par d'autres que son concepteur, fut, dès 1816 , un des décorateurs les plus appréciés de la scène théâtrale parisienne sous la Restauration ${ }^{2}$. Longtemps négligée, la création picturale et théâtrale de Daguerre a fait l'objet de plusieurs analyses récentes ; l'ouvrage de Stephen Pinson, dédié à toutes les facettes de son œuvre, permet désormais de disposer d'un outil de recension très précieux des dessins, des peintures, des projets de décor et des daguerréotypes attribués à Daguerre ${ }^{3}$. L'étude, érudite et clairvoyante, de Philippe Ortel des liens existants entre la photographie et la littérature, dès les prémices, a montré combien l'invention daguerrienne entretient des rapports étroits avec l'art littéraire et scénique de son temps ${ }^{4}$. Plus récemment, la restauration du dernier diorama de Daguerre, réalisée pour l'église de Bry-sur-Marne, petite ville à l'Est de Paris où il résidait, a fait l'objet d'une publication, qui serait à enrichir ${ }^{5}$. Ces différentes analyses, que l'exposition au musée d'Orsay avait catalysées, offre de poser un regard neuf sur l'invention photographique, souvent encore vue sous le seul registre de la technique ${ }^{6}$. La personnalité de l'un des deux inventeurs français éclaire les conditions de création, dans le contexte très riche du renouvellement des arts au début du XIXe siècle ${ }^{7}$.

Le daguerréotype a souvent été mis au regard avec la peinture; leurs similitudes de conception ont été mis en exergue - leur caractère d'œuvre unique, leur inscription dans un cadre, leur double soumission à la perspective albertienne - et leurs différences soulignées - l'utilisation d'une technique liée à la chimie et à l'alchimie, l'opposition de la surface réfléchissante du métal avec le velouté du papier ou le fini de la toile, l'apparition du contingent et du fortuit en contrepoint des nécessaires sacrifices que choisit de faire le peintre pour arrêter sa composition ${ }^{8}$. Les relations 
entre le daguerréotype et le théâtre de son temps s'articulent autour de trois postulats essentiels, que Daguerre plaça au cœur de sa création théâtrale comme de son invention photographique : le désir de ne pas rompre l'illusion de la représentation, l'utilisation de la lumière, le sens de la composition fondé sur un rapport des masses qui puise, en partie, sa logique dans les procédés de conception des décors théâtraux. Grâce à Daguerre, un des hommes de spectacle les plus doués de son temps, la photographie se conçut ainsi avec une théâtralité assumée. Elle composait, devant son objectif, une scène préalablement arrêtée. Philippe Ortel a souligné la capacité de la chambre obscure daguerrienne à transformer le monde en scène, à faire du réel une représentation: "Après tout, photographier n'est-il pas un geste scénographique à l'envers? Tandis qu'Hugo met le monde sur la scène, Daguerre invente avec Niépce la machine qui transforme en scène tous les lieux du monde enregistrables; le théâtre est une chambre noire et la chambre noire un théâtre ${ }^{9}$. " Par essence, la photographie se révèle ainsi théâtralisante, au sens strict.

Le théâtre rencontrait, au début du XIXe siècle, un succès et une effervescence qui en firent un des loisirs préférés des Parisiens. Bien que sévèrement encadrée par le système du privilège - promulgué en 1804 par Bonaparte et qui ne fut aboli qu'en 1864 - qui limitait le nombre des théâtres parisiens à quatorze et spécifiait la nature des œuvres que chaque lieu était autorisé à jouer, la scène théâtrale joua un rôle essentiel dans la création artistique de son temps. L'art théâtral connaissait alors un renouveau exceptionnel, dont Stendhal avait souligné la nécessité en 1822 : « La poésie dramatique en est en France au point où le célèbre David trouva la peinture en $1780^{10}$. » $\mathrm{Ce}$ renouveau portait sur la nature des œuvres jouées; l'introduction du drame shakespearien sur la scène française, en anglais, puis dans une traduction enfin fidèle à la nature des œuvres, rompait avec les règles de la tragédie classique. Il offrait ainsi les conditions de naissance du drame romantique et son double rapport au réel et au fantastique, à la représentation de la réalité comme à la création de l'illusion. La publication en 1833 du Paradoxe sur le comédien de Diderot, écrit dans les années 1780, s'inspirait des innovations de jeu mises en œuvre en Grande-Bretagne. Il n'y avait alors pas de metteur en scène : la représentation se réglait, sous le contrôle du directeur du théâtre - que les nécessités d'une bonne gestion désignaient comme un partenaire exigeant - entre l'auteur, l'acteur et le décorateur ${ }^{11}$. Le rôle de ce dernier était essentiel; les décors étaient attendus par les spectateurs et souvent applaudis pour eux-mêmes, ainsi que l'écrivit un critique en 1818: "Monsieur Daguerre est un auxiliaire bien dangereux pour un auteur; quand un ouvrage est couvert d'applaudissements, il tire à lui les trois-quarts de la couverture ${ }^{12}$. " Dans « Le parfait machiniste ", un des contes constituant les Kreisleriana de Hoffmann, paru en 1813, Maître Kreisler, le maître de chapelle sous lequel se cachait l'auteur lui-même, souligne son attachement aux décors : "Quand je dirigeais encore l'orchestre de l'Opéra de ...., ma fantaisie et le hasard me conduisaient souvent sur la scène, et je m'inquiétais fort de la décoration et de la machinerie ${ }^{13}$.»

L'époque était friande de spectacles d'optique, fondés sur la capacité de leurs concepteurs à parvenir à des effets d'illusion, suscitant l'émoi et la surprise des spectateurs. Le succès du panorama, inventé en 1788 à Edimbourg par Robert Barker mais qui ne connut nulle part ailleurs un succès plus grand et plus durable qu'à Paris, se déclinait à différentes échelles, du jeu pour enfants aux scènes gigantesques et somptueuses construites pour Pierre Prévost. La mode pervertit même, comme le souligna Balzac dans le Père Goriot, le langage. Pour être de leur temps, les 
pensionnaires de la pension Vauquer parlèrent en "rama ", ajoutant ce suffixe à tous leurs propos. Les procédés mis au point pour le panorama, portés par la double ambition d'une reproduction fidèle du réel et d'une recherche de l'illusion, les jeux d'éclairage qui les animaient comme les sujets retenus, liés à ce goût du sublime défini au milieu du XVIIIe siècle par Burke et soutenu par Diderot, furent repris et déclinés ensuite au théâtre. En 1930, Marie-Antoinette Allevy écrivait : "Il se dégagea dès l'origine, de ces spectacles qui n'étaient ni théâtre, ni peinture, mais qui se rattachaient si étroitement à ces deux arts, une conception toute particulière du monde extérieur. Monuments, sites pittoresques, manifestations les plus complexes de phénomènes naturels y étaient reproduits avec une exactitude qui faisait crier au miracle. Ces tableaux hybrides marquèrent d'une empreinte profonde d'abord l'art dramatique qui y puisa des sujets d'action scénique, ensuite, la décoration théâtrale chargée avant tout d'illustrer adroitement ces sujets. Les décorateurs du temps allaient, dans ces divertissements, trouver un inépuisable répertoire de modèles. $»^{14}$ La coïncidence s'étendait à la photographie; ce fut dans ce contexte curieux de découvertes optiques, friand d'effets lumineux, que s'inventa le nouveau procédé de reproduction du réel. En 1839, l'année même où François Arago présentait, devant l'Académie des Sciences de Paris puis devant la Chambre des députés, l'invention photographique de Daguerre, Théophile Gautier notait que «le temps des spectacles purement oculaires était venu ${ }^{15}$.»

En ouvrant, en juin 1822, rue Samson, en collaboration avec le peintre Charles Bouton (1781-1853), son Diorama, Daguerre exploitait, avec maestria, le goût de l'époque pour l'illusion et le trompe-l'œil. Il sut y mettre en œuvre ses qualités de décorateur et de peintre comme son énergie d'homme de spectacle. Il fit la preuve de son habileté à maitriser la lumière, rappelant qu'il avait été le premier, en 1820, à employer l'éclairage au gaz sur la scène de l'Académie de musique et de danse, pour la création d' Aladin et la lampe magique. Enthousiaste, Balzac écrivit à sa sœur Laure de Surville, en sortant du diorama, le 22 août 1822 : « Daguerre et Bouton ont étonné tout Paris; mille problèmes sont résolus depuis que, devant une toile tendue, on croit être dans une église à cent pas de la chose. C'est la merveille du siècle, une conquête de l'homme, à laquelle je ne m'attendais nullement ${ }^{16}$.» Ce registre de la surprise était celui de l'émotion, fondée sur l'émoi de la " terreur délicieuse » chère à Edmund Burke. Comme l'écrivit un critique parisien, après avoir vu La Vallée de Sarnen, un des premiers tableaux du diorama : «Le plus surprenant est le changement de lumière. D'un jour calme, doux, délicieux, serein, on passe à l'obscurité noire d'un orage qui se rapproche, une noirceur profonde et violente, tempétueuse, qui décolore chaque objet, qui nous fait guetter le tonnerre qui gronde dans le lointain et imaginer que nous sentons les larges gouttes, annonciatrices du déluge ${ }^{17}$."

La mission assignée au théâtre était de distraire mais surtout d'émouvoir. "Au théâtre, il faut rire, pleurer, s'effrayer, avoir peur, s'épouvanter " écrivit Hoffmann ${ }^{18}$. Hugo renchérit, dans la préface de Ruy Blas en 1838 : «La foule demande surtout au théâtre des sensations ; la femme des émotions, le penseur des méditations [...] le mélodrame pour la foule, pour les femmes, la tragédie qui analyse la passion, pour les penseurs, la comédie qui analyse l'humanité. » Le rôle du décor était essentiel dans cette émotion éprouvée; en maintenant l'illusion théâtrale, il parvenait à toucher l'âme du spectateur. "Tous deux [le décorateur et le machiniste] partaient de ce principe insensé que décorations et machines devaient servir le poème sans qu'on les remarquât, et que dès lors, grâce à l'effet d'ensemble, le spectateur devait se trouver 
transporté tout entier hors du théâtre, comme sur des ailes invisibles, dans le monde fantastique de la poésie. Ils étaient d'avis qu'il ne suffit pas, pour parfaire l'illusion, de décors combinés avec une profonde érudition ou un goût raffiné, des machines agissant avec une puissance enchantée et incompréhensible aux yeux du spectateur ", s'enthousiasma Hoffmann, imaginant le talent de Holbein, machiniste et décorateur au théâtre de Bamberg où séjourna le poète de 1808 à 1813, lors de l'écriture de ses contes ${ }^{19}$.

Goethe, en écrivant son premier Faust, dévoila son intention de séduire et émouvoir les foules. Sa référence fut scénographique, dévouant une place importante au décor supposé de la représentation : «C'est à la foule que je voudrais plaire! on vient ici au spectacle; on veut qu'il y ait beaucoup à voir. Si les yeux sont satisfaits, si vous présentez au public des tableaux variés et merveilleux, vous voguez à pleines voiles... Vous ne pouvez plaire à la foule que par la quantité... N'épargnez aujourd'hui ni décorations, ni machines ; faites paraître le soleil et la lune, semez des étoiles à pleines mains, usez à discrétion des eaux, des feux et des rochers, des bêtes féroces et des oiseaux de proie. Entassez entre ces quatre planches toutes les merveilles de la création, et percevez d'un seul coup d'œil les cieux, la terre et les enfers ${ }^{20} \ldots .$. » En 1828, le critique du Globe loua les qualités de la série de lithographies que le jeune Eugène Delacroix avait consacrées à Faust, série que Goethe, au soir de sa vie, considéra comme la meilleure interprétation de son œuvre. "Faust ne sera donc une fête que pour ceux qui aiment les surprises de machinerie, les apparitions et les disparitions sensationnelles, les monstres divins de la sorcellerie et toute la fantasmagorie du ciel et de l'enfer. Et qui n'aime pas cela aujourd'hui ?»

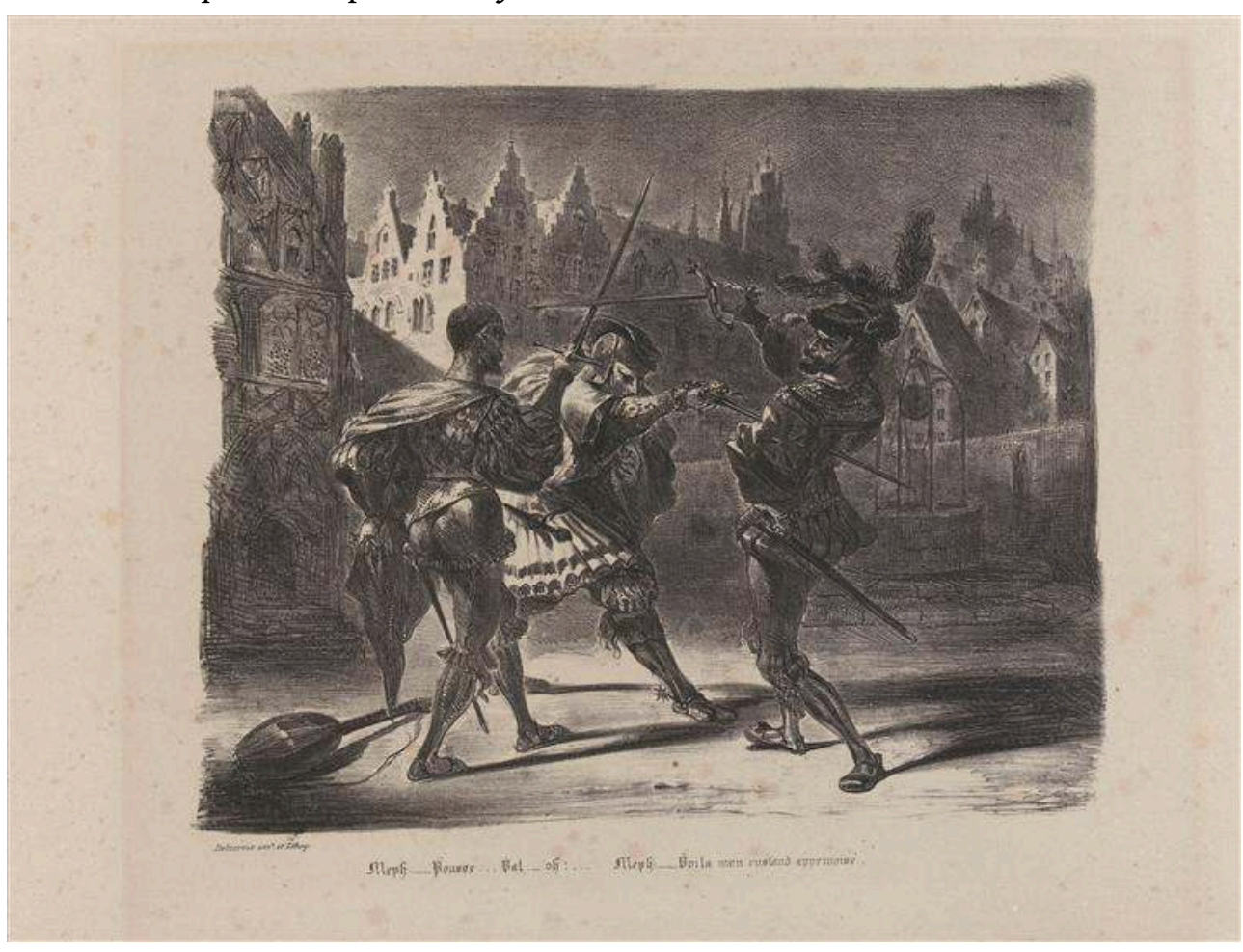

Eugène Delacroix, Duel de Faust et Valentin, 1827 (Paris, musée Eugène-Delacroix, MD 2002-52)

LITHOgRAPHIE

IMAgE FOURNIE PAR L'AUTEUR DE L'ARTICLE 
L'illusion théâtrale ne se fonde pas sur une exactitude objective; elle est une illusion librement consentie, et, comme telle, appréciée. Stendhal, dans le dialogue qu'il imagina entre l'Académicien et le Romantique, s'opposant sur les mérites comparés de Racine et de Shakespeare, souligna que l'illusion parfaite était un leurre exquis que l'auteur, les acteurs comme le décorateur devaient savoir offrir au public. Shakespeare, à ses yeux, le permettait mieux que Racine, le drame que la tragédie, grâce aux détails naïfs, au sens habile de la vérité. "Avoir des illusions, être dans l'illusion, signifie se tromper, à ce que dit le dictionnaire de l'Académie. Une illusion, dit M. Guizot, est l'effet d'une chose ou d'une idée qui nous déçoit par une apparence trompeuse. Illusion signifie donc l'action d'un homme qui croit la chose qui n'est pas, comme dans les rêves, par exemple. L'illusion théâtrale, ce sera l'action d'un homme qui croit véritablement existantes les choses qui se passent sur scène. [...] Les spectateurs savent bien qu'ils sont au théâtre et qu'ils assistent à la représentation d'un ouvrage d'art. Il est impossible que vous ne conveniez pas que l'illusion que ce que l'on vient chercher au théatre n'est pas une illusion parfaite. [...] Il me semble que ces moments d'illusion parfaite sont plus fréquents que l'on ne croit en général [...]. Mais ces moments durent infiniment peu. [...] Ces instants délicieux et si rares d'illusion parfaite ne peuvent se rencontrer que dans la chaleur d'une scène animée. [...] [T]out le plaisir que l'on trouve au spectacle tragique dépend de la fréquence de ces petits moments d'illusion, et de l'état d'émotion où, dans l'intervalle, ils laissent l'âme du spectateur ${ }^{21}$. "

L'illusion, pour Daguerre comme pour Stendhal, était d'autant plus vraie que l'émotion était profonde. Le réel vrai se distinguait ainsi du réel exact; la vraisemblance se nourrissait de la richesse d'une perception sensorielle. Daguerre sut dans son diorama, théâtre sans acteurs où l'intrigue tenait tout entière dans la magie des transformations du décor, et le jeu de scène dans la variation des lumières, faire naître cette émotion teintée à la fois de mélancolie et d'angoisse, mêlant rêve et réalité, qu'attendaient le public et la critique d'alors. Par la justesse et la précision de ses didascalies lumineuses, il parvint à atteindre une authenticité des détails et une vérité d'ensemble qui séduisit le public parisien. Décorateur de talent, peintre reconnu, Daguerre était au fait des débats théâtraux et picturaux de son temps, sensible aux enjeux de leur renouvellement. Les romans de Walter Scott, ses visions d'une Ecosse brumeuse et mélancolique, lui inspirèrent nombreuses de ses représentations. 


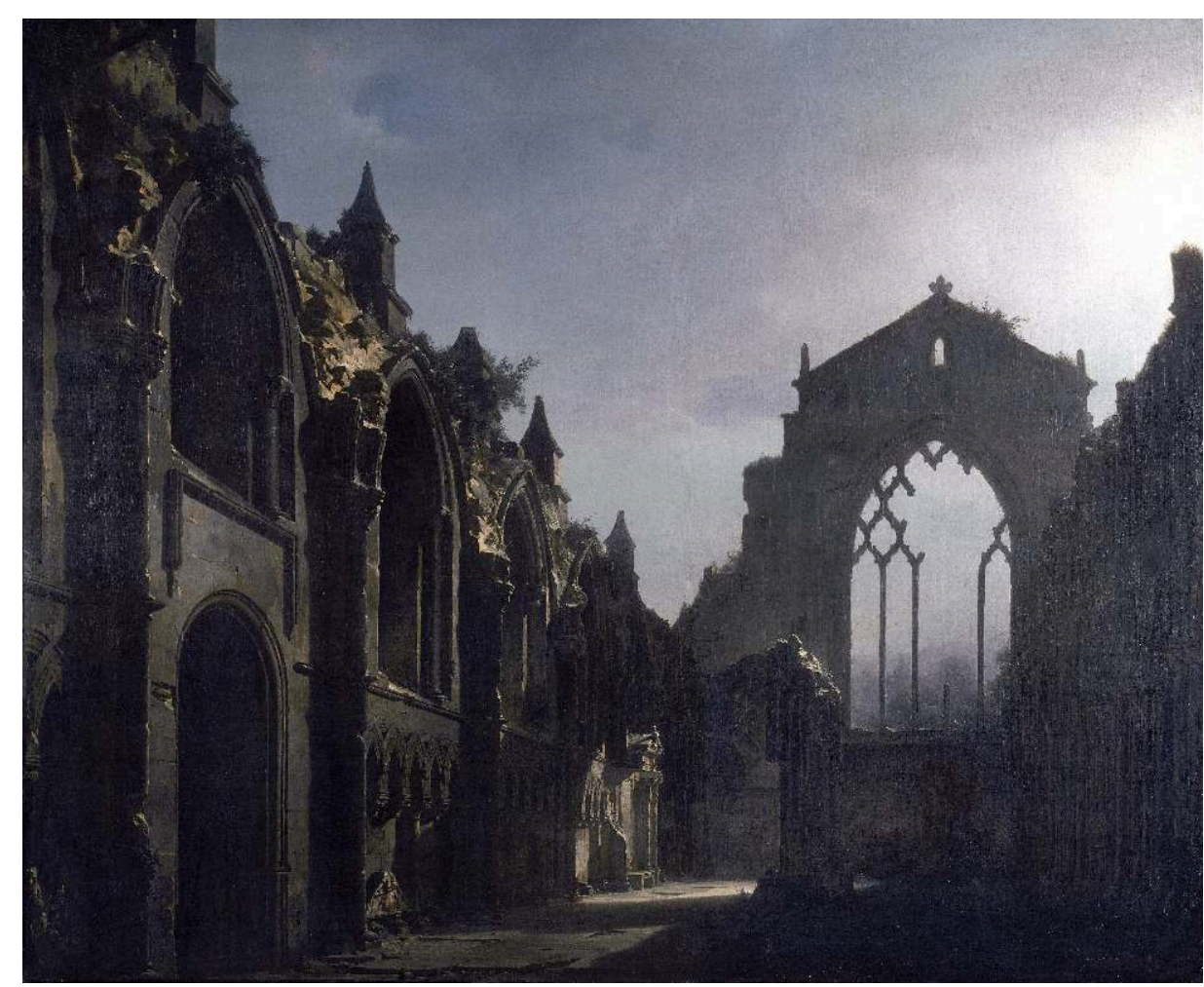

Daguerre, The Ruins of Holyrood Chapel, 1824 (Liverpool, Art Gallery)

Huile sur toile. URL : http://en.wikipedia.org/wiki/

File:The_Ruins_of_Holyrood_Chapel_(Louis_Daguerre),_1824_(Google_Art_Project).jpg

LICENCE WIKIMEDIA COMMONS, THE GOOgLE ART PROJECT

Il sut offrir à la lumière une puissance évocatrice, proche de celle recherchée alors par Delacroix, Léon Cogniet (1794-1880) ou le jeune Delaroche (1797-1856).

En 1834, Daguerre mit au point, avec l'assistance du peintre Hippolyte Sébron (1801-1879), élève de Léon Cogniet, un diorama «double effet», que Mentienne, le premier biographe de l'inventeur, définit ainsi : «Deux sujets étaient peints sur la même toile de chaque côté et c'est en éclairant cette toile par devant et ensuite par derrière que la première scène devenant invisible, laissait alors apparaitre la seconde. Là était le secret de l'invention ${ }^{22}$.» La Messe de minuit à Saint-Etienne du Mont rencontra un succès considérable; de nouveau le public parisien, la reine Marie-Amélie ellemême, se précipita au diorama. Un critique anonyme - peut être Jules Janin - fit ainsi, dans L'Artiste, l'éloge de Daguerre : « Vous montez un escalier obscur, et vous arrivez à tâtons dans un amphithéâtre. La nuit est complète. En face de vous, se détachent, sur un espace infini et silencieux, quelques grandes lignes architecturales de formes indécises [...] Peu à peu, la lumière s'approche et la foule se dessine, des galeries immenses s'éclairent, le temple lui-même apparait, magnifique, resplendissant, enveloppé des flots de lumière qui s'échappent d'un nuage éclatant et livrent à tous les regards l'aspect imposant de cette scène. [...] Aucune des expositions de M. Daguerre n'a été destinée comme celle-ci à frapper aussi vivement l'imagination et à obtenir un succès d'enthousiasme. Aussi, la foule se porte-t-elle, depuis quelques jours au Diorama [...] ». Népomucène Lemercier (1771-1840) publia un poème laudateur dans Le Journal des Débats: "Daguerre est sans égal dans son diorama, il dispose à son choix de chaque effet solaire [...]. De ce tableau vivant, l'illusion parfaite retient l'œil et l'esprit 
dans une erreur complète, mais l'aurore paraît et tout s'évanouit, le jour a remplacé la messe de minuit ${ }^{23}$."

Daguerre livra le secret de son invention dans sa Description des procédés de peinture et d'éclairage du diorama: «Bien que dans ces tableaux, il n'y ait effectivement de peints que deux effets, l'un de jour par devant, l'autre de nuit peint par derrière, ces effets ne passant de l'un à l'autre que par une combinaison compliquée des milieux que la lumière avait à traverser, donnaient une infinité d'autres effets semblables à ceux que présente la nature dans ses transitions du matin au soir et vice-versa ${ }^{24}$. » Il est difficile à l'historien moderne de juger justement de la portée finale de ces représentations; l'observateur contemporain est réduit à imaginer le spectacle et sa magie à partir des quelques toiles de Daguerre, d'un corpus de dessins et de gravures et des commentaires des critiques

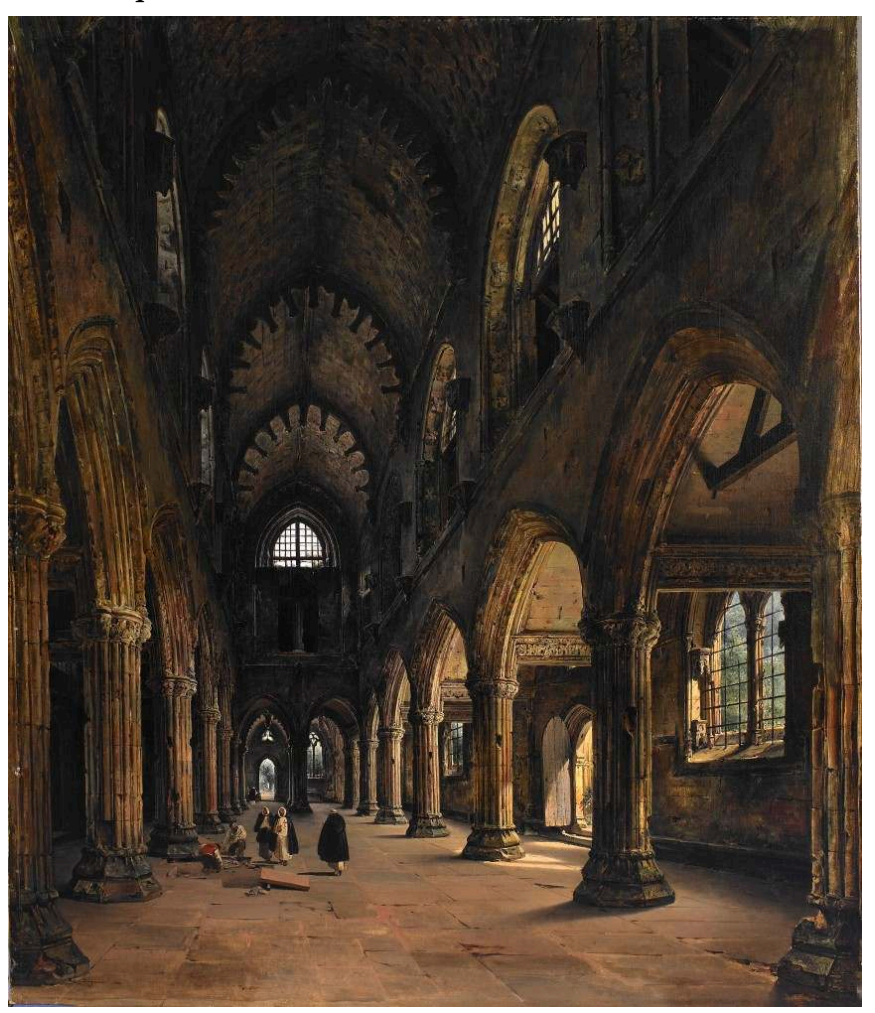

Daguerre, Intérieur de Rosslyn Chapel, 1824 (Rouen, Musée des Beaux-arts, inv. 2004.3.1)

\section{HUILE SUR TOILE, PRÉPARATOIRE À UN DÉCOR DE DIORAMA}

IMAgE FOURNIE PAR L'AUTEUR DE L'ARTICLE

Le succès qu'il rencontra dans les années 1820 et 1830 se comprend au regard d'un temps où les effets lumineux et de clair-obscur constituèrent non seulement un mode de représentation, mais aussi un cadre de pensée, pour les artistes, peintres, dramaturges et poètes.

Les liens entre les deux inventions majeures de Daguerre, le diorama et le daguerréotype, liens aujourd'hui le plus souvent oubliés, furent mis en exergue par Jules Janin dès son premier article consacré au procédé photographique dans L'Artiste ${ }^{25}$. L'un et l'autre, écrivit-il, sont le fruit de l'habileté de Daguerre à maîtriser la lumière et à la mettre à son service ; l'inventeur lui commandait de "façon impérieuse ", semblant en fixer lui-même les limites. Aux yeux de Janin, écrivain et critique de théâtre, 
diorama et daguerréotype étaient de même nature : ils constituaient un art au-delà de l'art, puisant dans leur maitrise de l'illusion la représentation de la réalité. Théodore de Banville, alors tout jeune poète, s'enthousiasma pareillement :

Que le spectre entrevu dans une chambre obscure

Se fixe sous mes mains et, de la terre au ciel

Prenant tous ses aspects à l'immense nature

La lumière sera le peintre universe ${ }^{26}$ !

Nicole Wild analysa récemment ces rapprochements: "La grande originalité des recherches de Daguerre réside dans la reproduction des phénomènes de la nature. [...] Passionné par les spectacles d'optique, il utilise des jeux de lumière mouvants afin d'obtenir une sorte de décor en mouvement, avec effets de brouillard, de nuages qui passent, de soleil qui se lève, de nuit qui s'estompe sous les effets de la lune ${ }^{27}$.» Les décors d'Elodie, pièce de Victor Ducange créée à l'Ambigu-Comique en 1822 furent, ainsi, particulièrement applaudis. "Rien n'est plus beau que les décorations faites par MM. Daguerre et Gosse ; l'effet est admirable et l'illusion complète » écrivit un critique du Miroir des Spectacles, le 12 janvier 1822. La vue intérieure d'un monastère, terrasse à demie noyée dans la pénombre et s'ouvrant au-delà d'une balustrade à claire-voie sur une toile de fond panoramique, offre un exemple caractéristique de la manière de Daguerre, mêlant ombre et lumière, associant la permanence du paysage et la fugacité de l'atmosphère.

Janin, non sans s'abandonner à un enthousiasme teinté d'utopie, s'extasiait de la possibilité offerte - théoriquement alors - par le daguerréotype de saisir l'instant et les transformations de l'atmosphère: "pas un de ces tableaux, exécutés d'après le même procédé, ne ressemble au tableau précédent: l'heure du jour, la couleur du ciel, la limpidité de l'air, la douce chaleur du printemps, la rude austérité de l'hiver, les teintes chaudes de l'automne, le reflet de l'eau transparente, tous les accidents de l'atmosphère se reproduisent merveilleusement dans ces tableaux merveilleux ${ }^{28}$ ». Humboldt lui fit écho dans son courrier à son ami le médecin et peintre Carl Gustav Carus (1789-1869) : «La lumière diffuse produit les mêmes effets que la lumière solaire. De belles reproductions des quais ou des vues du Paris lointain par temps de forte pluie. Gradation de la luminosité, le Palais et Jardin des Tuileries à cinq heures du matin, en été, à deux heures dans la chaleur de l'été et à sept heures lors du coucher du soleil, tout est saisi en une seule couleur monochrome ${ }^{29}$ ». 


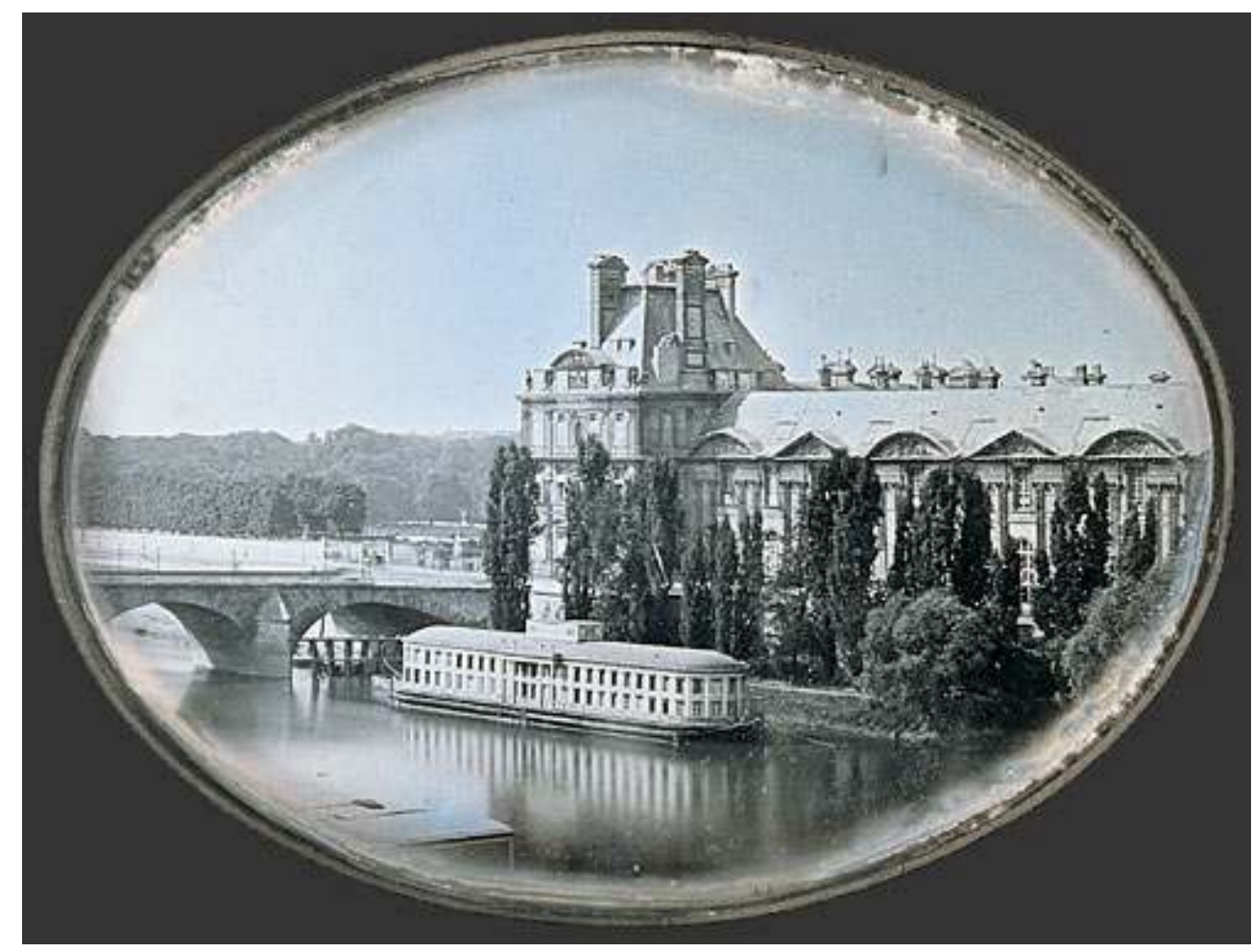

Daguerre, Palais du Louvre, pavillon de Flore, 1839 (Paris, musée des Arts et métiers)

IMAgE FOURNIE PAR L'AUTEUR DE L'ARTICLE

DAgUERRÉOTYPE, 16,2×21,3 CM

L'utilisation de la lumière et de l'ombre, sa rivale obscure, fut au cœur de la création romantique. Simone Delattre a montré comment se forma au début du XIXe siècle un genre littéraire autour du Paris de l'ombre ${ }^{30}$. Le genre échappait à l'espace littéraire pour devenir un mode d'observation de la ville et de ses monuments, proche de celui des premiers daguerréotypes de Daguerre. Ces vues diurnes, grâce au jeu de la lumière et des ombres, grâce aux effets des masses sombres des édifices, du reflet de l'eau font écho aux observations nocturnes de la ville: «La ville nocturne offre certes à la contemplation un décor intemporel, mais l'immuabilité des pierres ne résiste pas longtemps aux projections imaginaires : la nuit parisienne ressortit aussi à un exotisme de l'intérieur où l'organique, la barbarie, la conspiration, l'anomalie ont leur place. » Le demi-jour, le clair-obscur triomphaient sur la scène comme dans la gravure ou la peinture. Les effets de lumière mis en œuvre par Daguerre pour son spectacle Le Déluge présenté au Diorama à la fin des années 1820 trouvèrent un écho durable sur la scène comme en peinture. Les décors conçus par Cambon et Pilastre pour un spectacle donné au Cirque universel, Le Déluge universel ou l'Arche de Noé, d'Augustin Hapdé, reprirent les modes opératoires de Daguerre en matière d'éclairage, visant à obtenir le même effroi et saisissement dans l'âme du spectateur. Un critique de 1830 mit en évidence l'analogie avec le Diorama mais aussi avec les tableaux du peintre anglais John Martin : "L'étonnant tableau de la fin où l'on voit réunies les pensées de Poussin, de Daguerre, de Girodet et de Martin parait d'une prodigieuse vérité31. » 


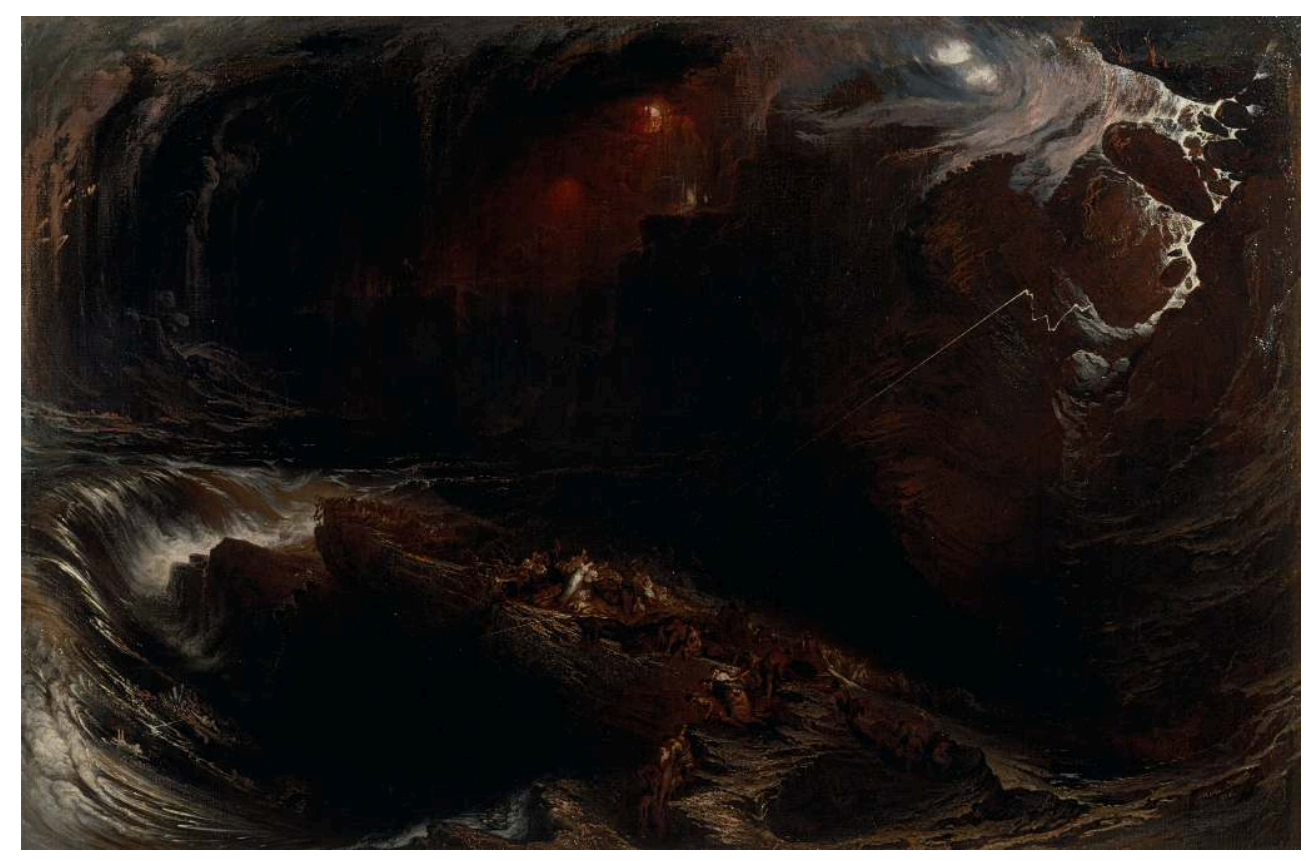

John Martin, Le Déluge, 1834 (Yale Center for British Art, Paul Mellon collection, inv. B 1978.43.11)

Huile sur toile, URL : http://upload.wikimedia.org/wikipedia/commons/2/26/John_Martin__The_Deluge_-_Google_Art_Project.jpg

LICENCE WIKIMEDIA COMMONS, GOOgLE ART PROJECT

Les œuvres de Martin comme celles de Daguerre jouaient sur les effets d'ensemble, sur la puissance de la lumière ; dans les années 1850, Thoré-Bürger écrivit ainsi : «Martin n'est pas un peintre. C'est une puissance mystérieuse qui n'a de rang, ni de place nulle part, qui se soucie peu de la forme de sa pensée, pourvu qu'il émeuve, qu'il étonne et qu'il galvanise la pensée d'autrui. Il se complait dans une poésie sans nom, embryonnaire, inachevée, confuse, qui excite l'imagination jusqu'à l'enivrement, mais qui ne laisse jamais dans l'âme du spectateur une impression complète et durable ${ }^{32}$. » Le grand succès de Scribe et de Cicéri, La Muette de Porticci, reprit dans la scène finale de l'éruption du Vésuve les effets du Diorama, de la mise en lumière et de la dramatisation souhaitée par Daguerre. Le célèbre tableau de Delacroix, L'Assassinat de l'évêque de Liège, illustrant un épisode du Quentin Durward de Walter Scott, usa, grâce au coup de lumière qui désigne le prélat et son meurtrier, les effets de lumière du théâtre. 


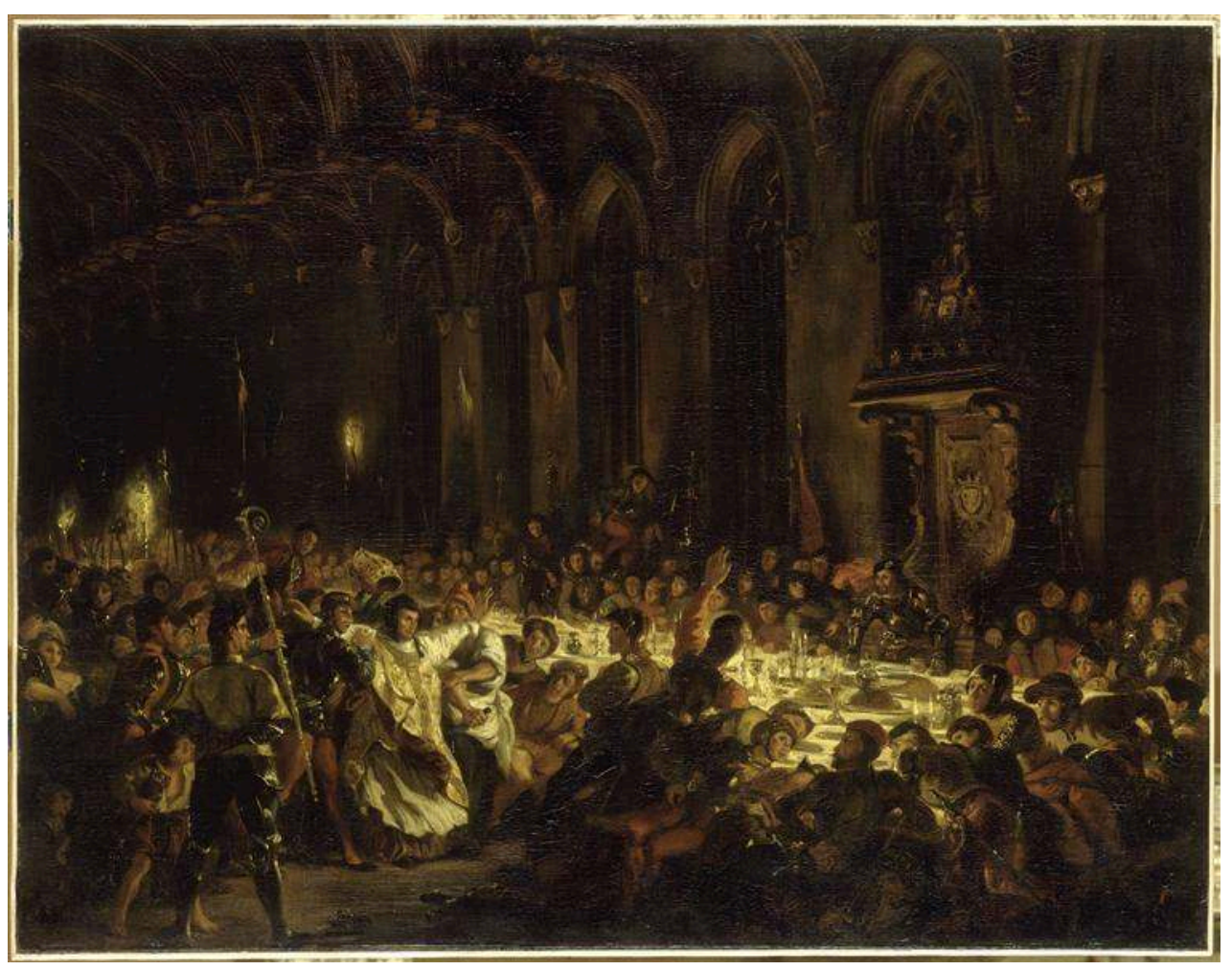

\section{Eugène Delacroix, L'Assassinat de l'évêque de Liège, 1829 (Paris, Louvre)}

URL : http://upload.wikimedia.org/wikipedia/commons/e/e8/Sanglier_des_Ardennes.jpg?uselang=fr LICENCE WIKIMEDIA COMMONS

Ces jeux d'ombre et de lumière devinrent, par leur force, partie intégrante du sujet. La même intensité prévaut dans les premières natures mortes reproduites par le daguerréotype. Les objets choisis pour la diversité de leurs formes, leur capacité à accrocher la lumière et à construire l'espace semblaient révélés par l'image daguerrienne, nimbés de mystère et de poésie. Humboldt apprécia tout particulièrement ces plaques: "L'effet le plus splendide est obtenu à l'aide de la lumière d'une lampe, éclairant des statues en marbre, des bas-reliefs en marbre. De telles plaques, longues de six pouces, ou plus grandes aussi, sont remarquables sous l'effet d'une lumière éblouissante ${ }^{33}$."

La camera obscura comme métaphore de l'esprit du poète devint un motif courant à l'époque romantique. Dans sa préface faussement postérieure aux Fantaisies à la mode de Callot de Hoffmann, Jean-Paul en fit déjà usage : «Sur les murs de sa [celle d'Hoffmann] chambre noire (camera obscura), et dans les couleurs les plus naturelles, les coquetants vibrions de la colle et du vinaigre se livrent à des mouvements vifs, s'affrontent, tourbillonnent avec des claquements secs. Sous une forme réduite, fruits d'une humeur facétieuse et parfaitement sarcastique, sont dépeints tout aussi bien les tristes jeux de l'amour et de l'art que les arts eux-mêmes sans oublier les amateurs d'art; le trait en est accusé, les couleurs chaudes, le tout empli d'âme et de libertés ${ }^{34}$.» Aloysius Bertrand dans Gaspard de la Nuit, premier poème en prose écrit en 1836 et paru, de façon posthume, en 1842, joua des analogies entre la création poétique et celle de la chambre noire; il multiplia les espaces entre ombre et lumière où, sous le mystère et le fantastique, la réalité vraie plus qu'exacte surgissait. Dans sa préface de l'édition de 
1980, Max Milner analysait: "L'espace des poèmes de Gaspard de la Nuit n'est pas seulement évidé, fragmenté, miniaturisé ; il est aussi traversé de lumières qui en élargissent les proportions, en dramatisent les perspectives, en rendent plus mystérieuses les surfaces et les profondeurs. [...] La nuit n'est pas seulement, chez Bertrand, un thème littéraire ou une expérience existentielle, comme chez la plupart des romantiques, mais une sorte de dispositif optique, commandant une suite mélodique de surgissements et d'évanescences dont le lecteur peut jouer avec une relative liberté35. " Dans " Maître Ogier, 1453 ", Bertrand place Charles VII face à une ouverture du Louvre dans la place qu'aurait pu avoir un photographe de la Monarchie de Juillet, l'œil collé à la chambre noire dans laquelle Paris tout entier apparaittrait : «Sire, demanda Maître Ogier au roi qui regardait par la petite fenêtre de son oratoire le vieux Paris éclairé d'un rayon de soleil, oyez-vous point s'ébattre, dans la cour de votre Louvre, ces passereaux gourmands, cette vigne rameuse et feuillue ${ }^{36}$ ? " Le Paris de la Monarchie de Juillet, avant les transformations d'Haussmann, si proche encore du Paris médiéval offrait la possibilité d'une telle analogie. Sainte-Beuve souligna la proximité des visions de Bertrand avec le daguerréotype: "de telles imagettes sont comme le produit du daguerréotype en littérature, avec la couleur en sus ${ }^{37}$ ».

Nul plus qu'Hugo n'a utilisé, comme cela a été mis en évidence par Philippe Ortel et Florence Naugrette, les analogies visuelles entre la puissance créative du poète et le pouvoir de réflexion et de concentration de la camera obscura. La préface de Cromwell, manifeste en 1827 de la toute jeune école romantique, multiplia ces exemples: «le drame est un miroir où se réfléchit la nature. Mais si ce miroir est un miroir ordinaire, une surface plane et unie, il ne renverra des objets qu'une image terne et sans relief, fidèle, mais décolorée; on sait ce que la couleur et la lumière perdent à la réflexion simple. Il faut donc que le drame soit un miroir de concentration qui, loin de les affaiblir, ramasse et condense les rayons colorants, qui fasse d'une lueur une lumière, d'une lumière une flamme. Alors seulement le drame est avoué de l'art. Le théâtre est un point d'optique ${ }^{38}$.» Hugo écrivit ces lignes au moment même où Daguerre, ayant appris les recherches de Nicéphore Niépce visant à fixer les images se formant dans la chambre noire, cherchait à le rencontrer. La coïncidence souligne combien la conception du daguerréotype, " cet art nouveau dans une vieille civilisation », comme le décrivit Gay-Lussac en 1839, était inscrite dans la création de son temps.

Ce fut sans doute au théâtre et à ses artifices que le daguerréotype dut son esthétique, mêlant réalité familière et étrangeté magique. L'utilisation de la camera obscura, celle des peintres d'architecture depuis le XVIe siècle, celle des décorateurs de panoramas et de théâtres en ce début du XIXe siècle privilégiait un point de fuite, une échappatoire $\mathrm{du}$ regard au centre d'une scène circonscrite par le cadre de l'image. La précision de l'image daguerrienne, sa clarté donnaient aux masses de l'architecture, un des premiers sujets choisis par Daguerre, une silhouette découpée, aux bords saillants, qui n'est pas sans évoquer celle des fermes de théâtre montées sur châssis, se tenant rigides sans appui, que l'inventeur utilisait pour ses décors à l'Ambigu-Comique. L'habileté des premiers daguerréotypistes à utiliser la lumière, à jouer sur ses variations progressives évoque, quant à elle, les variations lumineuses des décors scéniques. L'effet est particulièrement troublant dans la Vue du Palais Royal de Daguerre, conservée à Prague, où le quadrilatère de l'ancien Palais de Richelieu forme une scène bien délimitée. Le daguerréotype semble alors pleinement héritier des arts de l'illusion et du trompe-l'œil dont son inventeur fut un des maîtres les plus applaudis. 


\section{NOTES}

1. Critique citée par Alice et Helmut Gernsheim dans leur ouvrage sur Daguerre, L.J.M. Daguerre, The History of the Diorama and the Daguerreotype, $2^{\mathrm{e}}$ édition revue et corrigée, New York, Dover, 1868 , p. 59.

2. Je remercie très vivement Dominique Goy-Blanquet de m'avoir associée aux célébrations du 450e anniversaire de la naissance de William Shakespeare à Paris. L'exposition organisée au musée Eugène-Delacroix Eugène Delacroix, le fils le plus légitime de Shakespeare a été l'occasion de la présentation, dans leur ensemble, des pierres lithographiques de la suite Hamlet. Cet article s'appuie sur deux travaux antérieurs : «Le vrai sous le fantastique ", Etudes photographiques, $\mathrm{n}^{\circ} 16$, 2005, p. 152-165 ; «La photographie, avatar du romantisme », Romantismes et révolutions, II, actes du colloque de la Fondation des Treilles, Paris, Gallimard, 2008, p. 250-274.

3. Stephen Pinson, Speculating Daguerre, Art and Enterprise in the Work of J.L.M. Daguerre, Chicago et Londres, The University of Chicago Press, 2012.

4. Philippe Ortel, La littérature à l'ère de la photographie, Nîmes, Jacqueline Chambon, 2002

5. Guillaume Le Gall, La peinture mécanique, le diorama de Daguerre, Paris, Mare et Martin Arts, 2013.

6. Sous la direction de Quentin Bajac et de Dominique de Font-Réaulx, Le daguerréotype français, un objet photographique, Paris, Musée d'Orsay/RMN, 2003.

7. Daguerre s'associa, malgré leurs différences, avec Nicéphore Niépce en 1829 ; ce dernier avait, le premier, en 1826, réussi à fixer les images du réel grâce à la camera obscura. La France, on le sait, n'était pas le seul pays où des recherches s'effectuaient en ce sens. En Grande-Bretagne, William Henry Fox Talbot avait réussi, avec un procédé sur papier, à reproduire des éléments de sa création fin1838. A ce sujet, voir Michel Frizot (dir.), Nouvelle histoire de la photographie, Paris, Hachette, 1995, rééd. 2004 et Françoise Heilbrun (dir.), La photographie au musée d'Orsay, Paris, Musée d'Orsay/Flammarion, 2008.

8. Dominique de Font-Réaulx, Peinture et photographie, les enjeux d'une rencontre, Paris, Flammarion, 2012.

9. Philippe Ortel, op.cit., 2002, p.50.

10. Stendhal, Racine et Shakespeare, Paris, éd. Kimé, 1994, p. 19.

11. Florence Naugrette a montré l'apparition progressive du metteur en scène et le rôle essentiel joué alors par Alexandre Dumas et Victor Hugo dans la représentation de leurs pièces, Le théâtre romantique, Paris, Points/Seuil, 2002.

12. Journal des Débats, 1818.

13. "Le parfait machiniste" in Kreisleriana in Fantaisies à la manière de Callot, Paris, Phébus, Libretto, 2004, édition de 1979, p. 95-101.

14. M-A. Allevy, La mise en scène en France dans la première moitié du XIXe siècle, Paris, Droz, 1938, reprint Genève, Slatkine, 1976, p. 44.

15. Théophile Gautier, Histoire de l'art dramatique, Paris, 1869, tome II, p. 174-175.

16. Honoré de Balzac, lettre du 20 août 1822 à sa sœur, Laure de Surville, citée par Jacques et André Marie, "Une invention française : le diorama de Daguerre" in Une saison Lumière à Montpellier, Institut Jean Vigo, 1987, p. 179.

17. Cité par Georges Potonniée, Daguerre peintre et décorateur, Paris, Paul Mantel, 1935, p. 101.

18. Op. cit., p. 99.

19. Op. cit., p. 97.

20. Cité par M-A. Allevy, op. cit., p. 54.

21. Stendhal, Racine et Shakespeare, Paris, éd. Kimé, 1994, première parution en 1823, p. 22 et p. 23, c'est Stendhal lui-même qui souligne.

22. Cité par Jacques et Marie André, op.cit., p. 179. 
23. «Diorama, l'inauguration du Temple de Salomon » in L'Artiste, 1839, cité dans « Anthologie », Le daguerréotype français, un objet photographique, Paris, RMN, 2003, p.383.

24. Louis Jacques Mandé Daguerre, Description des procédés de peinture et d'éclairage du diorama, Paris, 1838, p. 79.

25. $1839,2^{\text {ème }}$ série, tome II, $11^{\mathrm{e}}$ livraison, p.145-147. Stephen Pinson souligne cette proximité entre Diorama et daguerréotype («Trompe l'œil: Photography's Illusion Reconsidered» in Nineteenth century Art Worldwide 1/1 [Spring, 2002]: http://www.19thc-artworldwide.org/ spring02/85-spring02/spring02article/195-trompe-loeil-photographys-illusion-reconsidered, consulté le 2 mars 2015).

26. Philippe Ortel, «Les doubles imaginaires de la photographie » in Romantisme, 1999, n¹05, p. 9-10. Le poème de Banville est paru en 1839.

27. Nicole Wild, "La scène " in L'invention du sentiment, Musée de la Musique, RMN/Cité de la Musique, 2002, p. 132-133.

28. Op.cit., p. 146.

29. Lettre datée du 25 février 1839 et publiée dans Le daguerréotype français, un objet photographique, Paris, Réunion des Musées nationaux, 2003, p. 385 et suivantes. La réduction des temps de pose fut la grande affaire de l'invention des photographes tout au long du XIXe siècle. Tous rêvèrent d'un procédé à même de reproduire l'instant et ses métamorphoses. Espoir vain et utopique, bien sûr. La photographie, comme l'a mis en exergue Roland Barthes, est toujours la représentation d'un moment arrêté, figé. Seul le cinéma permet de saisir ces évolutions du temps et de la matière. Au moment de l'invention du daguerréotype, les temps de pose sont encore très longs, autour de plusieurs minutes. A peine dix ans après, ils avaient, déjà, été réduits à quelques secondes.

30. Les douze heures noires, la nuit à Paris au XIXe siècle, Paris, Albin Michel, 2000, p.34.

31. Le Courrier des théâtres, 27 mai 1830, cité par M-A. Allevy, op.cit., p. 72.

32. Cité par Barthélémy Jobert, L'invention du sentiment, op.cit., cat. 61, p. 170-172.

33. Lettre du 25 fév. 1839 d'Alexandre von Humboldt, in Roland Recht, La Lettre De Humboldt : Du jardin paysager au daguerréotype, Paris, Christian Bourgois, 1969, p. 10.

34. Jean-Paul Richter, Préface des Fantaisies à la manière de Callot de E.T.A. Hoffmann, Bamberg, 1813 (daté par le préfacier de décembre 1823), Paris, Phébus, coll. Libretto, 2004, p.27.

35. Aloysius Bertrand, Gaspard de la Nuit, Paris, Gallimard, 1980

36. Op.cit., p. 159.

37. Cité par Ortel, op. cit., p.142.

38. Victor Hugo, Cromwell, Paris, Garnier-Flammarion, 1965, p. 90.

\section{RÉSUMÉS}

Par la figure de Jacques-Louis-Mandé Daguerre, l'un des inventeurs français, la photographie, dont l'apparition modifia profondément les codes de la représentation et le rapport au réel, est étroitement liée au renouveau théâtral du premier tiers du XIXe siècle. Daguerre, homme de spectacles, décorateur renommé au théâtre et à l'Opéra, fut, comme sur la scène, attaché à obtenir, grâce à l'image photographique, une illusion parfaite de la représentation. Son habileté à utiliser les sources de lumière lui fut précieuse pour la conception de son invention. Au-delà, la photographie est, par la nécessité d'une confrontation à l'objet ou à la scène reproduite, une 
« image performée », précédée obligatoirement d'une répétition du moment représenté. Cette communication souhaiterait rappeler ces origines théâtrales de la photographie, qui ont perduré longtemps dans la pratique photographique, en analysant également la part prise, dans les années 1820 et 1830, par l'intérêt renouvelé pour le théâtre et la littérature anglaise, celui de Shakespeare notamment, source d'iconographie nouvelle.

Photography, which has radically altered the codes of representation and the perception of reality, is closely linked to the renewal in drama that occurred in the first third of the 19th century, through the figure of Jacques-Louis-Mandé Daguerre, one of its French inventors. A famous set designer for the theatre and for the Opéra, Daguerre sought the perfect illusion in representation with photographic images as with his work for the stage. His clever use of light sources thus proved influential in the conception of his invention. Because photography implies a confrontation with the object or the scene reproduced, it is a kind of "performed image" which derives from a re-enactment of the moment represented. This article will go back to the origins of photography in theatrical practice, as well as analyse the importance of a renewed interest for English literature and drama, more specifically Shakespeare's, in the 1820s and 1830s, as a novel source of iconography.

\section{INDEX}

Keywords : Daguerre Louis, Delacroix Eugène, Hugo Victor, camera obscura, painting, photography, Shakespeare in France

Mots-clés : Daguerre Louis, Delacroix Eugène, Hugo Victor, camera obscura, peinture, photographie, Shakespeare en France

\section{AUTEUR}

\section{DOMINIQUE DE FONT-RÉAULX}

Conservateur général au Musée du Louvre, directrice du musée Eugène Delacroix 